\title{
Correction to: Myoclonic dystonia phenotype related to a novel calmodulin-binding transcription activator 1 sequence variant
}

\author{
Ivana Dzinovic ${ }^{1} \cdot$ Tereza Serranová $^{2} \cdot$ Clement Prouteau $^{3} \cdot$ Estelle Colin $^{3} \cdot$ Alban Ziegler $^{3} \cdot$ Juliane Winkelmann ${ }^{1,4,5,6}$. \\ Robert Jech ${ }^{2} \cdot$ Michael Zech ${ }^{1,4}$
}

Published online: 3 December 2021

(c) Springer-Verlag GmbH Germany, part of Springer Nature 2021

\section{Correction to: Neurogenetics}

https://doi.org/10.1007/s10048-021-00637-6

The affiliation of author Robert Jech was incorrectly indicated in the originally published version of this paper and should read: Department of Neurology, 1st Faculty of Medicine and General University Hospital in Prague, Charles University, Prague, Czech Republic. The affiliation appears correctly here and has been corrected in the paper online."

Publisher's note Springer Nature remains neutral with regard to jurisdictional claims in published maps and institutional affiliations.

The original article can be found online at https://doi.org/10.1007/ s10048-021-00637-6

Robert Jech

jech@cesnet.cz

$\bowtie$ Michael Zech

michael.zech@helmholtz-muenchen.de

1 Institute of Neurogenomics, Helmholtz Zentrum München, Munich, Germany

2 Department of Neurology, 1st Faculty of Medicine and General University Hospital in Prague, Charles University, Prague, Czech Republic

3 Department of Biochemistry and Genetics, University Hospital Angers, Angers, France

4 Institute of Human Genetics, Technical University of Munich, Munich, Germany

5 Lehrstuhl Für Neurogenetik, Technische Universität München, Munich, Germany

6 Munich Cluster for Systems Neurology, SyNergy, Munich, Germany 